\title{
The characteristics of gut microbiota and commensal Enterobacteriaceae isolates in tree shrew (Tupaia belangeri)
}

\author{
Wenpeng Gu',2, Pinfen Tong ${ }^{1}$, Chenxiu Liu', Wenguang Wang ${ }^{1}$, Caixia Lu', Yuanyuan Han', Xiaomei Sun',
} De Xuan Kuang ${ }^{1}$, Na Li ${ }^{1}$ and Jiejie Dai ${ }^{1 *}$ (D)

\begin{abstract}
Background: Tree shrew is a novel laboratory animal with specific characters for human disease researches in recent years. However, little is known about its characteristics of gut microbial community and intestinal commensal bacteria. In this study, $16 \mathrm{~S}$ rRNA sequencing method was used to illustrate the gut microbiota structure and commensal Enterobacteriaceae bacteria were isolated to demonstrate their features.

Results: The results showed Epsilonbacteraeota (30\%), Proteobacteria (25\%), Firmicutes (19\%), Fusobacteria (13\%), and Bacteroidetes (8\%) were the most abundant phyla in the gut of tree shrew. Campylobacteria, Campylobacterales, Helicobacteraceae and Helicobacter were the predominant abundance for class, order, family and genus levels respectively. The alpha diversity analysis showed statistical significance $(P<0.05)$ for operational taxonomic units (OTUs), the richness estimates, and diversity indices for age groups of tree shrew. Beta diversity revealed the significant difference $(P<0.05)$ between age groups, which showed high abundance of Epsilonbacteraeota and Spirochaetes in infant group, Proteobacteria in young group, Fusobacteria in middle group, and Firmicutes in senile group. The diversity of microbial community was increased followed by the aging process of this animal. $16 \mathrm{~S}$ rRNA gene functional prediction indicated that highly hot spots for infectious diseases, and neurodegenerative diseases in low age group of tree shrew (infant and young). The most isolated commensal Enterobacteriaceae bacteria from tree shrew were Proteus spp. (67\%) and Escherichia coli (25\%). Among these strains, the antibiotic resistant isolates were commonly found, and pulsed-field gel electrophoresis (PFGE) results of Proteus spp. indicated a high degree of similarity between isolates in the same age group, which was not observed for other bacteria.
\end{abstract}

Conclusions: In general, this study made understandings of the gut community structure and diversity of tree shrew.

Keywords: Tree shrew, Gut microbiota, Commensal Enterobacteriaceae, Susceptible age groups, Proteus spp.

\section{Background}

The tree shrew (Tupaia belangeri) is a small mammal similar in appearance to squirrel, widely distributed in South Asia, Southeast Asia and Southwest China [1]. Due to several specific characteristics, such as small adult body size, short reproductive and life cycle, low

\footnotetext{
*Correspondence: djj@imbcams.com.cn

'Center of Tree Shrew Germplasm Resources, Institute of Medical Biology, Chinese Academy of Medical Sciences and Peking Union Medical College, Yunnan Key Laboratory of Vaccine Research and Development on Severe Infectious Diseases, Yunnan Innovation Team of Standardization and Application Research in Tree Shrew, Zhao zong Road 66, Kunming 650118, China

Full list of author information is available at the end of the article
}

cost of maintenance, high brain-to-body mass ratio, and close affinity to primates, the tree shrew has been proposed as an alternative laboratory animal (nonhuman primate) in biomedical researches in recent years [2-4]. Currently, several studies have used this animal for human disease investigations, including hepatitis $C$ virus [5], and Epstein-Barr virus [6], as well as brain development and aging [7, 8], social stress and depression $[9,10]$. Although the biochemical metabolism, physiological function and genomic signature of tree shrew have been reported before [11-13], some important biological features are still unknown, for instance, the gut microbiota

(C) The Author(s). 2019 Open Access This article is distributed under the terms of the Creative Commons Attribution 4.0 International License (http://creativecommons.org/licenses/by/4.0/), which permits unrestricted use, distribution, and 
and commensal intestinal bacteria of this laboratory animal.

The gut microbial mutualisms, commensalisms, and pathogen interactions have been considered as important factors for animal health $[14,15]$. The composition and diversity of microbial community within and between host individuals are influenced by diet, life style, and disease [16, 17]. Furthermore, previous studies showed that characteristics of the host, such as gender or age, were responsible for variation in the gastrointestinal microbiome $[18,19]$. Up to present, a large number of studies on gut metagenomics by using next generation sequencing have been reported, including human, domestic or wild animals $[15,20]$, seldom referred to laboratory animal, especially for tree shrew. On account of its unified diet or life cycle in the laboratory feeding environment, the gut microbiota changes maybe more related with gender or age features of tree shrew. In addition, Enterobacteriaceae are the large Gram-negative bacteria, comprised of over 50 genera and 210 species. The members of this family are widely distributed across different ecological niches, including the environment, plants, and animals [21]. The majority of Enterobacteriaceae in the gut are considered commensals, as they perform beneficial for the host; however, some are considered as important pathogens in the setting of public health, such as pathogenic Escherichia, Salmonella spp., Yersinia spp. and Shigella spp. [21]. So far, there is no systemic research on intestinal commensal bacteria in tree shrew, specifically for Enterobacteriaceae. The identification of commensals or pathogenic bacteria from the gastrointestinal tract of this animal will provide the baseline for future human diarrhea disease researches. In this study, 16S rRNAtargeted amplicon sequencing method was used to investigate the gut microbiota of tree shrew, and Enterobacteriaceae strains were isolated to identify the characteristics of bacteria.

\section{Results}

\section{Taxonomic of the tree shrew gut microbiota}

For the 60 fecal samples, 4,167,908 reads were obtained from tree shrew, and 4,057,554 valid reads were acquired after merging and quality trimming. The average length of amplicon was $407.52 \pm 4.19 \mathrm{nt}$, ranged from 401 to $420 \mathrm{nt}$. The Q30 of sequencing was above $95 \%$ for all the samples, and $\mathrm{GC} \%$ was $50.78 \% \pm 0.85 \%$. In total, 5880 OTUs were found in the database, Epsilonbacteraeota (30\%), Proteobacteria (25\%), Firmicutes (19\%), Fusobacteria (13\%), and Bacteroidetes $(8 \%)$ were the most abundant bacterial communities at the phylum level; at the class level, Campylobacteria (30\%), Gammaproteobacteria (24\%), Fusobacteriia (13\%), Clostridia (9\%) and Bacteroidia (8\%) were the major microbiota;
Campylobacterales (30\%), Aeromonadales (19\%), Fusobacteriales (13\%), Clostridiales (9\%) and Bacteroidales (8\%) were the top five at order level; the top five at family level were Helicobacteraceae (25\%), Succinivibrionaceae (18\%), Fusobacteriaceae (13\%), Bacteroidaceae (6\%), and Lachnospiraceae (5\%); finally, Helicobacter (25\%), Anaerobiospirillum (18\%), Fusobacterium (13\%), Bacteroides (6\%), and Campylobacter (4\%) were the primary microbial communities at genus level, as shown in Fig. 1a. However, the relative abundance of gut microbiota for each sample was quite different, as shown in Fig. $1 \mathrm{~b}$ and $\mathrm{C}$. The higher relative abundance of Epsilonbacteraeota were found in sample tree shrew 28 (TS28), TS34, TS37, TS38 and TS40, but lower in TS7, TS22, TS23, TS64 and TS66; TS7, TS22 and TS70 had higher abundance for Proteobacteria, but lower for TS37, TS38 and TS40. At the genus level, similar results were identified, such as TS19, TS28 and TS38 rich in Helicobacter; TS7, TS22, and TS72 in Anaerobiospirillum. According to the gender of the tree shrew, 5065 OTUs were obtained in male group, compared with 4489 in female, and more OTUs were found in male group (Fig. 1d). For the age groups, the numbers of OTUs were increased with the aging of tree shrew, as shown in Fig. 1e. One hundred and sixty two unique OTUs were discovered in infant group, 208 in young, 404 in middle and 1071 in senile group.

\section{Diversity analysis}

The alpha diversity estimations showed that numbers of OTUs, Chao1, Shannon entropy were statistical different $(P<0.05)$ for age groups of tree shrew, indicated the significant diversity between four age groups. The OTU numbers and diversity were increased followed by the aging process. However, no statistics $(P>0.05)$ were found for gender groups of all the alpha diversity indexes except the numbers of OTUs in this animal (Table 1).

Beta diversity analyses were performed according to gender and age grouping. PCoA plot based on weighted and unweighted Fast UniFrac distance metric revealed two clustering gender groups were generated; however, large numbers of male and female samples were mixed together and cross connected from two cycles shown in Fig. 2a and b. The UPGMA dendrogram of gender groups showed two clusters in Fig. 2c (yellow and blue areas); each cluster also contained mix male and female tree shrew samples. The Anosim analysis indicated no statistical significance between male and female groups $(\mathrm{R}=0.02, P=0.156)$, as shown in Fig. 2d. For the age groups of tree shrew, two obvious clustering groups were found between infant and senile (Fig. 2e and f), while the young and middle were randomized distributed in PCoA plot. The UPGMA dendrogram of age groups also showed two clusters (Fig. 2g); the majority of red cluster area was senile group samples, parts of the middle group samples were located in this cluster, such as 


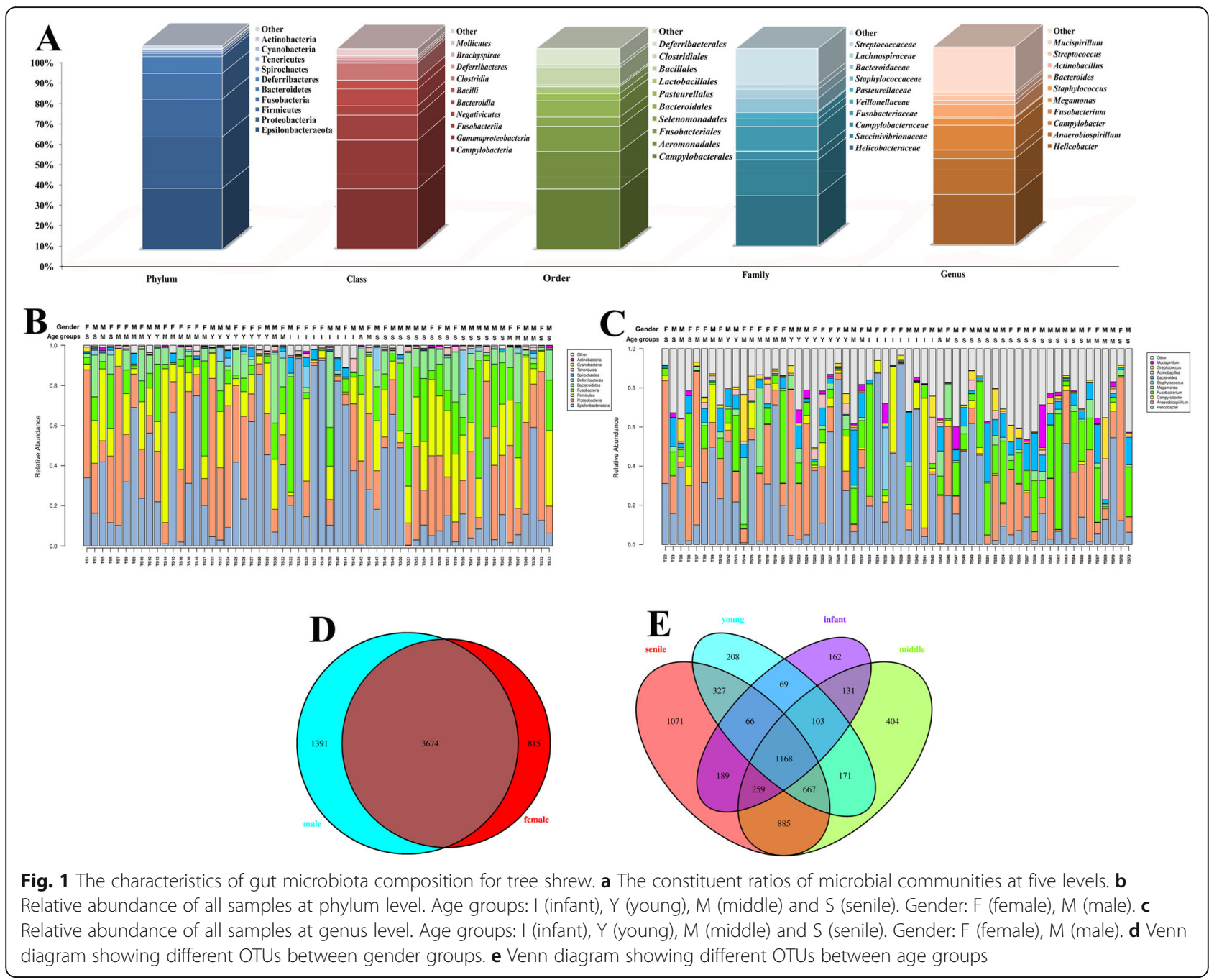

Table 1 The alpha diversity estimation of sequencing results in this study

\begin{tabular}{|c|c|c|c|c|c|}
\hline \multirow[t]{2}{*}{ Variables } & \multirow[t]{2}{*}{ Groups } & \multicolumn{4}{|c|}{ Indexes (mean \pm STD) } \\
\hline & & Numbers of OTUs & Chaol & Shannon entropy & Simpson's index \\
\hline \multirow[t]{2}{*}{ Gender } & Male & $763.90 \pm 183.57$ & $1415.49 \pm 358.88$ & $4.25 \pm 0.95$ & $0.85 \pm 0.11$ \\
\hline & Female & $658.03 \pm 217.81$ & $1290.31 \pm 412.13$ & $3.97 \pm 1.18$ & $0.78 \pm 0.16$ \\
\hline T-test & & 2.04 & 1.96 & 1.85 & 1.91 \\
\hline$P$ value & & 0.046 & 0.058 & 0.079 & 0.061 \\
\hline \multirow[t]{4}{*}{ Age groups } & Infant & $477.44 \pm 224.85^{\mathrm{a}}$ & $828.44 \pm 375.96^{\mathrm{a}}$ & $3.19 \pm 1.23^{b}$ & $0.72 \pm 0.19$ \\
\hline & Young & $697.10 \pm 147.22$ & $1263.44 \pm 280.52$ & $4.10 \pm 1.01$ & $0.80 \pm 0.17$ \\
\hline & Middle & $719.78 \pm 184.83$ & $1318.21 \pm 356.67$ & $4.19 \pm 1.05$ & $0.83 \pm 0.12$ \\
\hline & Senile & $806.09 \pm 168.54$ & $1503.51 \pm 330.79$ & $4.57 \pm 0.93$ & $0.85 \pm 0.11$ \\
\hline F (ANOVA) & & 7.26 & 8.65 & 3.91 & 2.04 \\
\hline$P$ value & & 0.000 & 0.000 & 0.013 & 0.118 \\
\hline
\end{tabular}

athe numbers of OTUs and Chao1 indexes of infant group had statistical significance $(P<0.05)$ with both young, middle and senile groups. No significant difference was found between young, middle and senile groups.

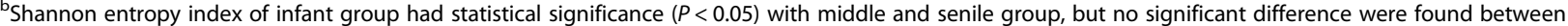
young, middle and senile groups 

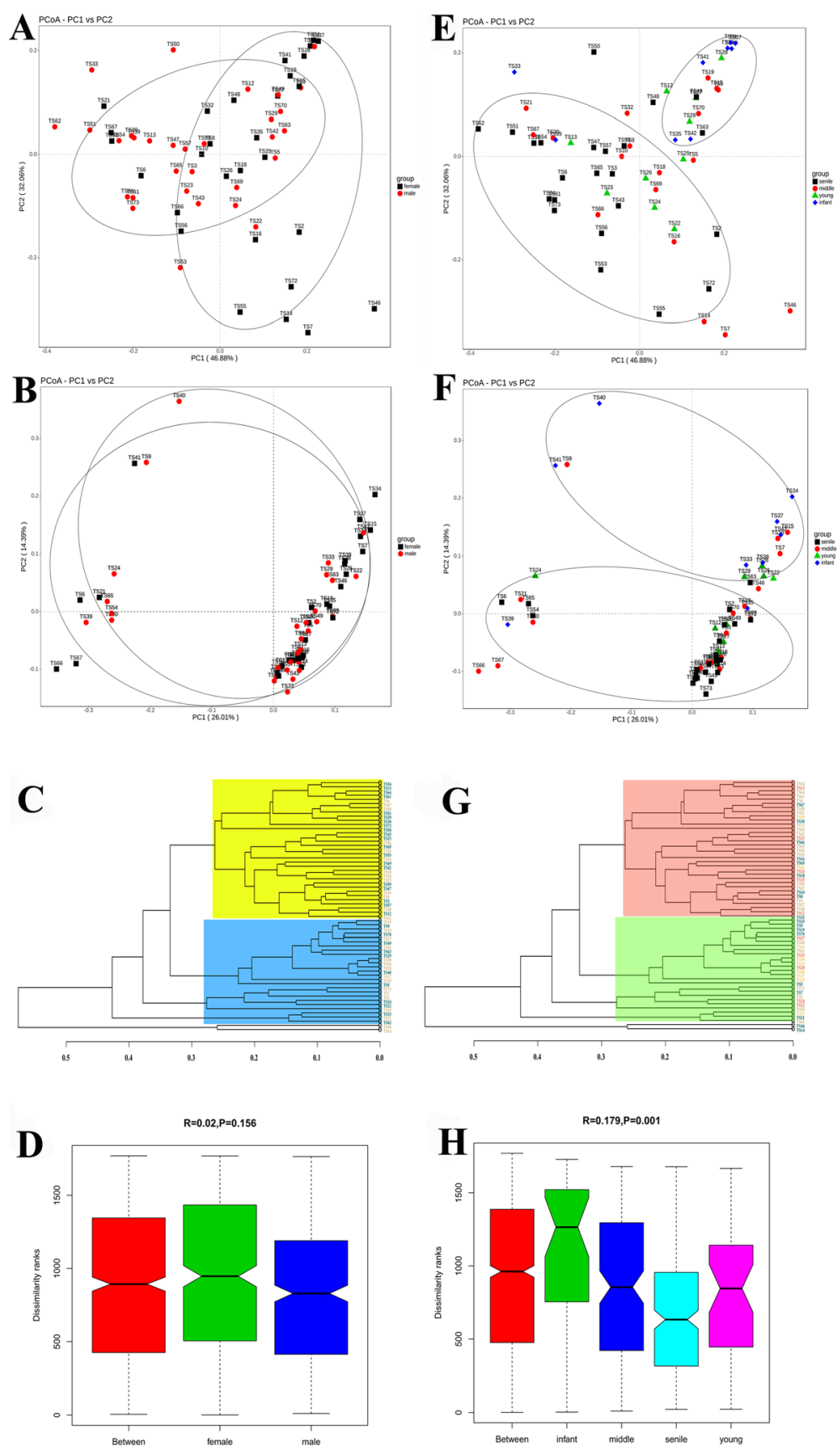

Fig. 2 Beta diversity analysis of tree shrew fecal samples. a PCoA plot based on weighted unifrac distance of the male and female group. b PCoA plot based on unweighted unifrac distance of the male and female group. c UPGMA dendrogram of gender groups for all the samples. Blue samples indicated male, and pink represented female. $\mathbf{d}$ Anosim analysis between male and female group. e PCoA plot based on weighted unifrac distance of the four age groups. $\mathbf{f}$ PCOA plot based on unweighted unifrac distance of the four age groups. $\mathbf{g}$ UPGMA dendrogram of age groups for all the samples. Yellow samples were infant group; reds were young group; blues were middle group; pinks were senile group. $\mathbf{h}$ Anosim analysis between infant, young, middle and senile group

TS67, TS30, and TS66. The green cluster area in Fig. $2 \mathrm{~g}$ contained most infant group samples, young and middle group samples were found in this cluster as well. The Anosim statistic revealed significant difference among age groups $(\mathrm{R}=0.179, P=0.001)$, as shown in Fig. $2 \mathrm{~h}$.

\section{Variation analysis}

LEfSe analyses were performed on significant differences of microbial taxa in abundance among tree shrew age and gender groups. The LDA score $\geq 3.0$ of gut microbiota between four age groups were shown in Fig. 3a. 


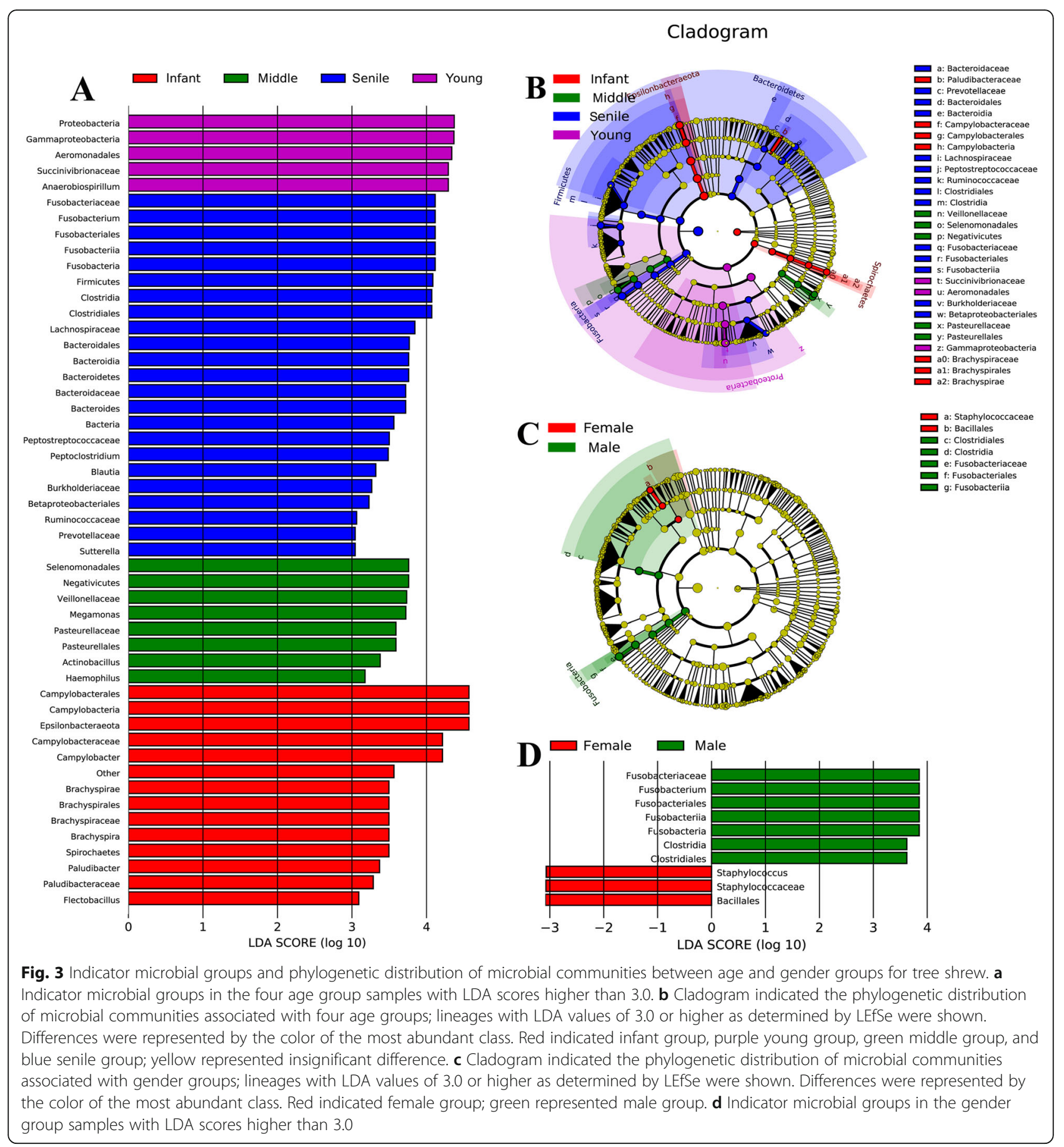

There were thirteen microbial taxa enriched in the infant group, five microbial taxa enriched in the young group, eight microbial taxa enriched in the middle group, and twenty-three enriched in the senile group. The most different abundant microbial taxa in infant group were Epsilonbacteraeota (the phylum), Spirochaetes (the phylum), Campylobacteria (the class), Brachyspirae (the class), Campylobacterales (the order), Brachyspirales (the order), Campylobacteraceae (the family) and Brachyspiraceae (the family). Proteobacteria (the phylum), Gammaproteobacteria (the class), Aeromonadales (the order) and Succinivibrionaceae (the family) were the primary taxa in young group. Meanwhile, Negativicutes (the class), Selenomonadales (the order), Pasteurellales (the order) and Pasteurellaceae (the family) were significantly enriched in middle group. Firmicutes (the phylum), Fusobacteria (the phylum), Bacteroidetes (the phylum), Clostridia (the class), Fusobacteriia (the class), Bacteroidia (the class), Clostridiales (the order), 
Fusobacteriales (the order), Bacteroidales (the order), Fusobacteriaceae (the family) and Bacteroidaceae (the family) were the representative taxa in the senile group. Cladogram showed the phylogenetic distribution of dominant classified microbial taxa associated with the age groups (Fig. 3b). The biomarkers of significant differences in abundance between male and female were also showed in Fig. 3c. Cladogram indicated that Clostridia (the class), Fusobacteriia (the class), Clostridiales (the order), Fusobacteriales (the order) and Fusobacteriaceae (the family) were enriched in male group, while Bacillales (the order) and Staphylococcaceae (the family) were predominant in female group. The gut microbial taxa presented statistically significant differences with an LDA threshold $\geq 3.0$ between male and female group were shown in Fig. 3d.

KEGG pathway annotation results based on PICRUSt revealed organismal systems, cellular process and human diseases concentrated on infant and young age groups, while the genetic information processing, metabolism and environmental information processing for senile group (Additional file 1). The metabolic pathways analysis showed higher hot spots for cell growth and death, infectious diseases, translation, genetic information processing, energy metabolism, neurodegenerative diseases, cell motility and environmental adaptation in low age group of tree shrew (infant and young); metabolism, membrane transport, endocrine system, carbohydrate metabolism, replication and repair were higher in senile age group, as Additional file 2 shown. The details of annotation information for metabolic pathway among four age groups were shown in Additional file 3. However, the annotation results according to gender groups had no such trends of concentration for gene functional predictions, as shown in Additional files 4, 5, 6.

\section{Characteristics of isolated Enterobacteriaceae bacteria}

One hundred and five strains were isolated from 73 tree shrew feces; among them, 28 samples had multiple species isolated. Proteus spp. (71, 67\%) and E. coli (25, 25\%) were the most Enterobacteriaceae bacteria (Fig. 4a). For Proteus spp., the majority of species was $P$. mirabilis (68, 96\%), and only three $P$. vulgaris $(4 \%)$ were isolated. All the entero-pathogenic bacteria, including Vibrio spp., Salmonella spp., and Shigella spp. etc. were not found in this study, including diarrheogenic $E$. coli by multiplex PCR. The Proteus spp. isolated results had no statistical significance with gender $(\mathrm{H}=0.01, P=0.922)$ and age groups $(\mathrm{H}=0.348, P=0.555)$ of tree shrew. The antibiotic resistant results showed high resistant rate for Oxacillin (OX) (100\%), Erythromycin (E) (100\%) and Tetracycline (TE) $(94.40 \%), 12$ isolates $(16.90 \%)$ were identified as extended-spectrum $\beta$-lactamases strains (ESBL) (Table 2). The gender of tree shrew $(\mathrm{H}=7.774, P=0.005)$ and species of Proteus spp. $(\mathrm{H}=15.184, P=0.000)$ showed the

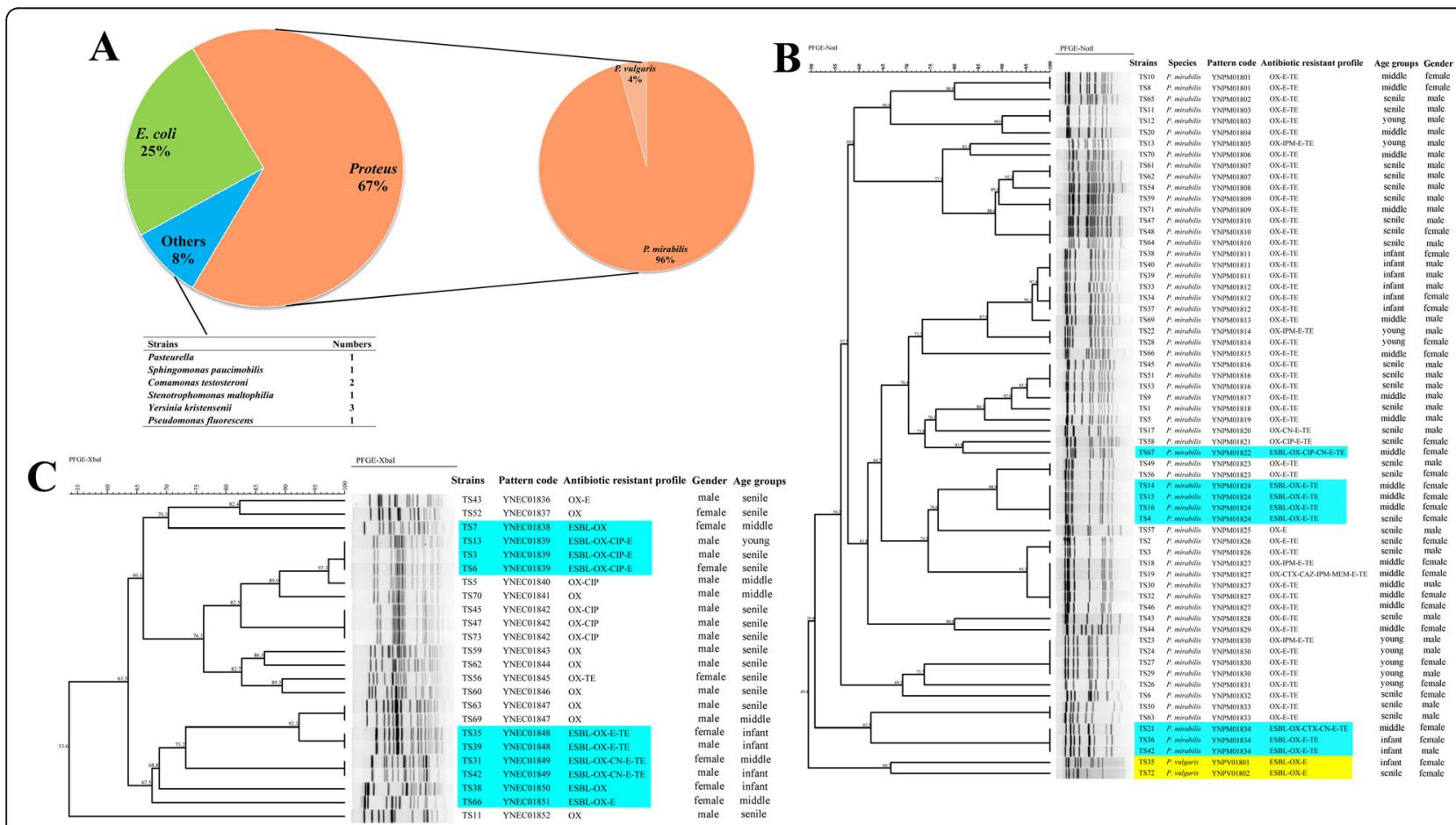

Fig. 4 The characteristics of isolated Enterobacteriaceae bacteria from tree shrew. a The isolation results of fecal samples for tree shrew. $\mathbf{b}$ PFGE cluster results of 71 Proteus spp. in this study. The yellow area was P. vulgaris strains, blue area indicated ESBL isolates for P. mirabilis. c PFGE cluster results of $25 \mathrm{E}$. coli in this study. Blue area indicated ESBL isolates for E. coli 
Table 2 The antibiotics resistant results of isolated Enterobacteriaceae bacteria in this study

\begin{tabular}{|c|c|c|c|c|c|}
\hline \multirow[t]{2}{*}{ Antibiotics } & \multirow[t]{2}{*}{ Interpret } & \multicolumn{2}{|l|}{ Proteus spp. } & \multicolumn{2}{|l|}{ E.coli } \\
\hline & & Strain numbers & Percent (\%) & Strain numbers & Percent (\%) \\
\hline \multirow[t]{2}{*}{ ESBL } & Resistant (R) & 12 & $16.90 \%$ & 10 & $40.00 \%$ \\
\hline & Sensitive (S) & 59 & $83.10 \%$ & 15 & $60.00 \%$ \\
\hline \multirow[t]{2}{*}{ OX } & $\mathrm{R}$ & 71 & $100.00 \%$ & 25 & $100.00 \%$ \\
\hline & $S$ & - & - & - & - \\
\hline \multirow[t]{2}{*}{ CTX } & $\mathrm{R}$ & 2 & $2.80 \%$ & - & - \\
\hline & S & 69 & $97.20 \%$ & 25 & $100.00 \%$ \\
\hline \multirow[t]{3}{*}{ CAZ } & $\mathrm{R}$ & 1 & $1.40 \%$ & - & - \\
\hline & S & 69 & $97.20 \%$ & 25 & $100.00 \%$ \\
\hline & Intermediate (I) & 1 & $1.40 \%$ & - & - \\
\hline \multirow[t]{3}{*}{ IPM } & $\mathrm{R}$ & 8 & $11.30 \%$ & - & - \\
\hline & S & 49 & $69.00 \%$ & 25 & $100.00 \%$ \\
\hline & I & 14 & $19.70 \%$ & - & - \\
\hline \multirow[t]{2}{*}{ MEM } & $\mathrm{R}$ & 1 & $1.40 \%$ & - & - \\
\hline & $S$ & 70 & $98.60 \%$ & 25 & $100.00 \%$ \\
\hline \multirow[t]{3}{*}{ CIP } & $\mathrm{R}$ & 2 & $2.80 \%$ & 8 & $32.00 \%$ \\
\hline & S & 69 & $97.20 \%$ & 15 & $60.00 \%$ \\
\hline & I & - & - & 2 & $8.00 \%$ \\
\hline \multirow[t]{2}{*}{$\mathrm{CN}$} & $\mathrm{R}$ & 3 & $4.20 \%$ & 2 & $8.00 \%$ \\
\hline & S & 68 & $95.80 \%$ & 23 & $92.00 \%$ \\
\hline \multirow[t]{2}{*}{ AK } & $\mathrm{R}$ & - & - & - & - \\
\hline & $S$ & 71 & $100.00 \%$ & 25 & $100.00 \%$ \\
\hline \multirow[t]{3}{*}{ E } & $\mathrm{R}$ & 71 & $100.00 \%$ & 9 & $36.00 \%$ \\
\hline & $S$ & - & - & 13 & $52.00 \%$ \\
\hline & I & - & - & 3 & $12.00 \%$ \\
\hline \multirow[t]{2}{*}{ TE } & $\mathrm{R}$ & 67 & $94.40 \%$ & 6 & $24.00 \%$ \\
\hline & $S$ & 4 & $5.60 \%$ & 19 & $76.00 \%$ \\
\hline
\end{tabular}

statistical difference with ESBL strains. 83.30\% ESBL Proteus spp. were isolated from female tree shrew, compared with $16.70 \%$ in male group; and all the three $P$. vulgaris were the ESBL, but $13.20 \%$ for $P$. mirabilis. The Cefotaxime (CTX), Ceftazidime (CAZ), Meropenem (MEM), Ciprofloxacin (CIP), Gentamicin $(\mathrm{CN})$ and TE resistant results had no statistical significance $(P>0.05)$ with gender and age groups. Thirty six PFGE patterns were identified for all the Proteus spp. strains, showing a high degree of polymorphism (Fig. 4b), and two species of Proteus spp. were divided into two cluster groups. The PFGE patterns had significant difference $(\mathrm{H}=55.273, P=0.009)$ with age groups of tree shrew, indicated the highly similarity between isolates in the same age group, such as YNPM01811 and YNPM01812 in infant group; YNPM01830 in young group; YNPM01824 and YNPM01827 in middle group; YNPM01810 in senile group, as shown in Fig. 4b.

The E. coli isolation results also had no statistical significance with gender $(\mathrm{H}=3.202, P=0.074)$ and age groups $(\mathrm{H}=1.422, P=0.233)$ of tree shrew. Ten ESBL $E$. coli strains $(40 \%)$ were found in this study, and high antibiotic resistant rates were for OX (100\%), E (36\%), CIP (32\%), and TE (24\%) (Table 2). The gender $(\mathrm{H}=$ $5.765, P=0.016)$ and age groups $(\mathrm{H}=11.082, P=0.001)$ showed the statistical difference with ESBL E. coli strains. Sixty percent of the ESBL were found in female tree shrew, and $40.0 \%$ for male. Furthermore, half of the ESBL strains were isolated from infant group. Similar results could be obtained for TE with gender $(\mathrm{H}=4.186$, $P=0.041)$ and age groups $(\mathrm{H}=7.412, P=0.006)$ of this laboratory animal. The CIP, CN, and E resistant results had no statistical significance $(P>0.05)$ with gender and age groups. Seventeen PFGE patterns were found for all the E. coli strains, showing no statistical difference $(P>$ 0.05 ) with gender or age groups (Fig. 4c).

The antimicrobial resistant-associated genes were showed in Table 3. TEM and CTX-M were both detected for Proteus spp. and E. coli ESBL strains, among 
Table 3 The antimicrobial resistant-associated genes detected in this study

\begin{tabular}{|c|c|c|c|c|c|}
\hline \multirow{2}{*}{$\begin{array}{l}\text { Resistant } \\
\text { antibiotic }\end{array}$} & \multirow[t]{2}{*}{ Genes } & \multicolumn{2}{|c|}{ Proteus spp. } & \multicolumn{2}{|l|}{ E. coli } \\
\hline & & Numbers & Percent (\%) & Numbers & Percent (\%) \\
\hline \multirow[t]{3}{*}{$\overline{E S B L}$} & TEM & 5 & $41.67 \%$ & 8 & $80.00 \%$ \\
\hline & CTX-M & 3 & $25.00 \%$ & 2 & $20.00 \%$ \\
\hline & Unknown & 4 & $33.33 \%$ & - & - \\
\hline CTX and CAZ & Unknown & 2 & $100.00 \%$ & - & - \\
\hline IPM & NDM1 & 8 & $100.00 \%$ & - & - \\
\hline \multirow[t]{3}{*}{ CIP } & gnrA & - & - & 3 & $37.50 \%$ \\
\hline & qnrB & 2 & $100.00 \%$ & 4 & $50.00 \%$ \\
\hline & anrs & - & - & 1 & $12.50 \%$ \\
\hline \multirow[t]{2}{*}{$\mathrm{CN}$} & aadAl & 1 & $33.33 \%$ & - & - \\
\hline & aacA4 & 2 & $66.67 \%$ & 2 & $100.00 \%$ \\
\hline \multirow[t]{4}{*}{ E } & ereA & 39 & $54.93 \%$ & 1 & $11.11 \%$ \\
\hline & ereB & 12 & $16.90 \%$ & 3 & $33.33 \%$ \\
\hline & mphA & 4 & $5.63 \%$ & 3 & $33.33 \%$ \\
\hline & Unknown & 16 & $22.54 \%$ & 2 & $22.23 \%$ \\
\hline \multirow[t]{4}{*}{ TE } & tetA & 3 & $4.48 \%$ & 6 & $100.00 \%$ \\
\hline & tetB & 5 & $7.46 \%$ & - & - \\
\hline & tetE & 47 & $70.15 \%$ & - & - \\
\hline & tetO & 12 & $17.91 \%$ & - & - \\
\hline
\end{tabular}

them, TEM was the major resistant gene. All of the Proteus spp. resistant to Imipenem (IPM) had NDM1, and most of CIP resistant gene was $q n r B$ for tree shrew Enterobacteriaceae strains. The most resistant-associated genes for erythromycin of Proteus spp. were ereA (54.93\%) and ereB (16.90\%), but tetE $(70.15 \%)$ and tetO (17.91\%) for tetracycline (Table 3).

\section{Discussion}

Comparative genome analysis between tree shrew and human revealed that there was a high sequence identity for genes/pathways involved in infectious diseases and neuropsychiatric disorders [11]. The proteomics of muscle and liver tissues for tree shrew indicated that almost half of the proteins were highly similar to those of human [22]. Besides, at the neurophysiological or neuroanatomical levels, a close homology between tree shrew and human in the area of visual cortex was also found [23]. All these pieces of evidence have laid the foundation for using the tree shrew to study human related diseases [24]. Therefore, study on the characteristics of tree shrew gut microbiota could provide us the better understandings of the baseline for tree shrew used as a laboratory model.

The mammalian intestinal tract has large numbers of bacteria, playing an important role in host metabolism, immunity, nutrition, and behaviors. The numbers of microorganisms in the gut exceed the host cells and the functions encoded by gut microbiota greatly surpass those of the host [25]. Since the widely use of next generation sequencing techniques, several studies have substantially increased our knowledge for both host-associated and environmental microbial communities. Previous study [20] showed gut microbiota at higher taxonomic levels among 60 mammalian species, and most referred to Firmicutes (65.7\%) and Bacteroidetes (16.3\%), dominated out of 75 known microbial phyla. These phyla were originally shown to compose the majority of sampled human gut-associated phylotypes. The other phyla represented were the Proteobacteria (8.8\%), Actinobacteria (4.7\%), and Verrucomicrobia (2.2\%) etc. These results were in accordance with that the ancestor of amniotes possessed the microbiomes mostly comprised Firmicutes and Bacteroidetes [26]. Our previous research on migrated gulls also indicated Firmicutes and Proteobacteria were the most abundance phyla of this wild animal [27]. However, in this study, Epsilonbacteraeota was the most abundance phylum level for tree shrew, followed by Proteobacteria, and Firmicutes; furthermore, the Helicobacter and Campylobacter were the important microbial communities at genus level, belonged to Epsilonbacteraeota. In the past, Epsilonproteobacteria was the fifth validly described class of the phylum Proteobacteria. Waite et al. [28] reassigned this class to a novel phylum for propose the name Epsilonbacteraeota (phyl. nov.) based on 
assessment of nearly 300 phylogenetic tree topologies in 2017. It was very interesting that Epsilonbacteraeota was the most gut microbiota for tree shrew, especially for infant group. Some studies reported Helicobacter and Campylobacter species colonized the intestinal tract of many domestic animals, and zoo mammals. Goto et al. [29] found the current status of Helicobacter contamination in laboratory mice, rats, gerbils, and house musk shrews, the $66.7 \%$ colonies of $H$. suncus were detected in shrew. Whary et al. [30] revealed the naturally acquired Helicobacter infections in commonly used laboratory rodent species, including mice, rats, gerbils, and hamsters. Consequently, it was not surprised that Epsilonbacteraeota was the majority of microbial community for tree shrew; however, the results of Helicobacter infection of laboratory animals should be paid attention for further animal model investigation.

Several studies have exemplified the role that the intestinal microbe played in mammalian physiology, human health and disease $[15,17]$. The lack of balanced and healthy gut microbiota has been linked to susceptibility to infection, decreased lymphocyte and intestinal macrophage proliferation of the hosts [31]. However, these associations have not to be investigated in depth in nonhuman primates. The nonhuman primates were the most biologically related research animal models for human, and a better understanding of the gut microbial communities would provide the opportunity to evaluate the influence in nonhuman primate evolution and ecology [25]. To date, seldom studies referred to the relationship between gender and age with gut microbiota composition. In our study, we found the aging of tree shrew was significantly responsible for variation of the microbial communities; the gut microbiota diversity was increased followed by the aging process of this laboratory animal, and for each age group, there were some representative bacteria. Amato et al. [18] determined that adult males, adult females, and juveniles have distinct microbiome compositions of black howler monkey, and juvenile and adult howlers possibly obtained nutritional benefits from the intestinal microbiome for their growth and reproduction. Ren et al. [19] found that wild yellow baboons possessed two different microbiome configurations, and determined that host age, diet and rainfall, were largely responsible for variation in the gastrointestinal microbiome. The tree shrew used in this study was closed population, the diet and feeding conditions were identical for the entire animal. Therefore, we considered that aging process was really responsible for variation of gut microbiota for tree shrew.

None of the intestinal pathogenic bacteria was detected in this study, among them; Enterobacteriaceae was the most one, especially for Proteus spp. and E. coli. Gordon et al. [32] analyzed 642 mammalian hosts for their isolated Enterobacteriaceae bacteria in Australia, and their results showed E. coli was the most common of the 24 enteric species. In our study, few species of Enterobacteriaceae bacteria was isolated compared with the wild migrated bird of our previous research [27], which possibly due to the single feeding environment or life cycle of tree shrew. Many wild and domestic animals, such as mammals, birds, reptiles, and insects were the hosts of Proteus spp. bacteria [33]. The relations between Proteus spp. with their hosts were still sometimes not determined. In our study, the isolated Proteus spp. was more likely to be the commensal, since no diseases or symptoms appeared in all these tree shrews. However, the antimicrobial resistant Enterobacteriaceae bacteria and related genes were detected among these strains, especially for erythromycin, tetracycline and $\beta$-lactamase, indicated the highly antibiotic resistant isolates were commonly existed in this laboratory animal.

\section{Conclusions}

As an alternative laboratory animal, tree shrew became widely used for human disease studies recently. In this study, we analyzed the gut microbiota structure and commensal Enterobacteriaceae bacteria for tree shrew. Significant diversity of microbial community was found between each sample, and the diversity was increased followed by the aging of this laboratory animal. The most isolated commensal Enterobacteriaceae bacteria were Proteus spp. and E. coli. Among these strains, the antibiotic resistant isolates were commonly found. In general, this study made understandings of the gut community structure and diversity of tree shrew.

\section{Methods}

\section{Sample collections and DNA extraction}

Seventy-three tree shrew fecal samples were collected at the Center of Tree Shrew Germplasm Resources, Institute of Medical Biology, Chinese Academy of Medical Science and Peking Union Medical College in Kunming, China. The tree shrews were closed population, and healthy without visible signs of tumors or disease, 39 were male, and 34 were female. The average age was $35.55 \pm 22.76$ months, ranged from 2 months to 75 months. We divided these animals into four age groups according to the previous study with some modification $[1,12]$; the infant group was under 7 months, young group was aged between 8 to 18 months, middle group was 19 to 42 months, and over 43 months was defined as senile. All of the tree shrews used in this study were the first filial generation, weighing $138.67 \pm 20.36$ g. Each tree shrew was housed in independent sterilized stainless steel cage containing hygienic food and water. The commercial full-price nutritive pellet was used for feeding twice a day, and the clean apple was fed once a week. Fresh fecal samples were collected and 
stored at $-80^{\circ} \mathrm{C}$ until processing all samples together for gut microbiota analysis. Each fecal sample was handled for two ways, one was isolated the commensal Enterobacteriaceae bacteria by using fresh feces, another was selected 60 samples to extract the genomic DNA for 16S rRNA sequencing. The total genomic DNA was extracted by using fecal sample's DNA extraction kit (Tiangen, Beijing) following the manufacturer's instructions. All the DNA samples were stored at $-20^{\circ} \mathrm{C}$ until usage.

\section{PCR amplication, library construction and sequencing}

The 16S rRNA gene ranged from V3 to V4 variable region was used as the target for bacterial community investigation by Illumina Miseq sequencing. PCR amplication primer was used according to Klindworth et al. [34] study and the protocal of library preparation guideline of Illumina. In general, PCR was performed by using KAPA HiFi HotStart ReadyMix kit (Kapa, Biosystems). Each PCR reaction contained genomic DNA $2.5 \mu$, forward and reverse primers $5 \mu \mathrm{l}$ respectively, and KAPA mixture $12.5 \mu$. The amplication procedure was based on our previous study, and then the products were purified with AMPure XP magnetic beads (Beckman, Coulter), quantified using Qubit fluorometer (Invitrogen, Life Technologies). The secondary PCR amplication was performed to add the Illumina Nextera barcodes, using i5 and i7 primers following the manufacturer's instruction, and then the purification process was executed again to remove nontarget fragments. Finally, the amplicons were normalized, pooled and sequencing was conducted using Illumina Miseq sequencing system (Illumina, SanDiego, USA).

\section{Bioinformatics and statistics}

The raw data were trimmed for quality check and filtered of low quality $(<\mathrm{Q} 25)$ reads. The paired end reads were merged to generate tags by using CLC Genomics Workbench 9.5.2 (QIAGEN, Denmark) [27]. The combinations of software QIIME (version 2) [35], USEARCH (version 11) [36] and R package (version 3.2) [37] were used for bioinformatics analysis. The merged tags were filtered by QIIME, and all the sequences were clustered into operational taxonomic units (OTUs), according to 97\% sequence similarity against Silva 132 database [38] using the UPARSE pipeline (http://drive5.com/usearch/ manual/uparsecmds). OTUs were named based on the genus level using SILVA taxonomic nomenclature.

Principal co-ordinates analysis (PCoA) was performed to visualize the similarities between samples for gender and age groups according to Bray-Curtis using Ape package. Anosim (Analysis of similarities) was used to compare the microbial composition difference between groups, and the statistical significant group $(P<0.05)$ was analyzed by LEfSe (Linear discriminant analysis
Effect Size) to identify the biomarker bacteria between groups ( $P$ value cutoffs, 0.05). PICRUSt (Phylogenetic investigation of communities by reconstruction of unobserved states) [39] was used to predict the functional contents from 16S rRNA gene through KEGG pathway database. Statistical analysis was performed by using SPSS software package (version 16.0, IBM, USA). Kolmogorov-Smirnov, T-test, ANOVA or Kruskal-Wallis $\mathrm{H}$ test were used if appropriate. $\mathrm{P}$ value of $<0.05$ was recognized as statistical significance. Sequence data were deposited on the NCBI database by the SRA accession: SRP151653.

\section{Isolation of Enterobacteriaceae bacteria}

The intestinal Enterobacteriaceae bacteria were isolated based on previous study [27]. All the fecal samples were inoculated on MacConkey Agar and Xylose Lysine Desoxycholate (XLD) agar (Luqiao, Beijing), incubated at $37^{\circ} \mathrm{C}$ for $24 \mathrm{~h}$. Selenite Brilliant Green Broth (SBG) and Buffered Peptone Water (BPW) (Luqiao, Beijing) were used as enrichment broth to isolate the Salmonella spp. and Vibrio spp., then the enrichments were inoculated on Salmonella Shigella agar (SS) and Thiosulfate citrate bile salts sucrose agar (TCBS) (Luqiao, Beijing), incubated at $37^{\circ} \mathrm{C}$ for $24 \mathrm{~h}$. Yersinia spp. was isolated according to wang et al. [40] method. All the suspected Enterobacteriaceae bacteria were picked and identified by using Vitek Compact 2 biochemical identification system (bioMérieux). In addition, all the isolated E. coli were detected using multiplex PCR diagnostic kit (ABTechnology, Beijing) for the diarrheogenic E. coli (DEC). The workflow for Enterobacteriaceae isolation and identification in this study was shown in Additional file 7.

\section{Antibiotic resistant test and genes detection}

All the isolates were performed antibiotic resistant test by broth micro-dilution method using customized microtiter plates (Sensititre, UK) according to the manufacturers' instructions. The minimum inhibitory concentrations (MICs) for 12 antibiotics was determined, Amoxicillin (AML), Amoxicillin/Clavulanic acid (AMC), Oxacillin (OX), Cefotaxime (CTX), Ceftazidime (CAZ), Imipenem (IPM), Meropenem (MEM), Ciprofloxacin (CIP), Gentamicin (CN), Amikacin (AK), Erythromycin (E), and Tetracycline (TE). The tests were interpreted in accordance with the Clinical and Laboratory Standards Institute (CLSI) guidelines (M100-S25, 2015); E. coli ATCC 25922 was used as quality control. The breakpoints of MIC values for Enterobacteriaceae bacteria were shown in Additional file 8. Antimicrobial resistant-associated genes were detected by PCR and sequenced using the primers based on previous studies [41-45]. The primers for different resistant genes were 
shown in Additional file 9. The bacterial genomic DNA was extracted by bacteria genomic DNA extraction kit (Tiangen, Beijing). The PCR reaction was performed in $20 \mu \mathrm{l}$ volume, contained $10 \mu \mathrm{l}$ Premix Taq (TaKaRa, Japan), $8 \mu \mathrm{l}$ water, $0.5 \mu \mathrm{l}$ each primers, and $1 \mu \mathrm{l}$ sample DNA. The amplification procedures were $94{ }^{\circ} \mathrm{C} 5 \mathrm{~min}$, followed by 30 cycles: $94{ }^{\circ} \mathrm{C} 15 \mathrm{~s}, 55^{\circ} \mathrm{C} 30 \mathrm{~s}, 72^{\circ} \mathrm{C} 30 \mathrm{~s}$, and finally $72{ }^{\circ} \mathrm{C} 10 \mathrm{~min}$. The amplified products were detected in $1.5 \%$ agarose gel. The positive amplicons were sent for bidirectional sequencing by TaKaRa, Japan.

\section{Pulsed-field gel electrophoresis (PFGE)}

PFGE was performed for isolated Proteus spp. and E. coli strains according to previous researches [46, 47], each plug was digested with NotI (TaKaRa, Japan) for Proteus spp. and XbaI (TaKaRa, Japan) for E. coli. CHEF-Mapper (Bio-Rad, USA) was used for electrophoresis, and the pulse time ranged from $5 \mathrm{~s}$ to $40 \mathrm{~s}$ (Proteus spp.) and $6.76 \mathrm{~s}$ to $35.38 \mathrm{~s}$ (E. coli) for $19 \mathrm{~h}$. The gels were stained with Gel-Red (Biotium) and visualized by using gel imaging system (Bio-Rad, Gel DocXR). PFGE patterns were analyzed using BioNumerics version 6.6, and dendrograms were constructed using the Dice coefficient and un-weighted pair group methods with the arithmetic mean algorithm (UPGMA).

\section{Additional files}

Additional file 1: Heatmap of KEGG pathway annotation results of age groups based on PICRUSt (level 1). (PDF $792 \mathrm{~kb}$ )

Additional file 2: Heatmap of the metabolic pathways results of age groups (level 2). (PDF $1231 \mathrm{~kb}$ )

Additional file 3: Heatmap of the detailed annotation information for metabolic pathway among four age groups (level 3). (PDF $1096 \mathrm{~kb}$ )

Additional file 4: Heatmap of KEGG pathway annotation results of gender groups based on PICRUSt (level 1). (PDF $795 \mathrm{~kb}$ )

Additional file 5: Heatmap of the metabolic pathways results of gender groups (level 2). (PDF $1021 \mathrm{~kb}$ )

Additional file 6: Heatmap of the detailed annotation information for metabolic pathway among gender groups (level 3). (PDF 1016 kb)

Additional file 7: The workflow for Enterobacteriaceae isolation and identification in this study. (PDF $1115 \mathrm{~kb}$ )

Additional file 8: The breakpoints of MIC values for Enterobacteriaceae bacteria in this study. (PDF $102 \mathrm{~kb}$ )

Additional file 9: The PCR primers for different resistant genes in this study. (PDF $359 \mathrm{~kb}$ )

\section{Abbreviations}

LEfSe: Linear discriminant analysis Effect Size; OTUs: Operational taxonomic units; PCoA: Principal co-ordinates analysis; PFGE: Pulsed field gel electrophoresis; PICRUSt: Phylogenetic investigation of communities by reconstruction of unobserved states

\section{Authors' contributions}

$\mathrm{JD}$ and XS designed the study. Experiments and laboratory analyses were performed by WG, PT, CLiu, WW, and NL. Analyses were performed by CLu, YH and DK. WG wrote the paper. (CLiu correspond to the author furthest up on the author list). All authors read and approved the final manuscript.

\section{Funding}

This work was supported by Yunnan Science and Technology Talent and Platform Program (2017HC019); Yunnan Joint Support for National Program (2015GA009); Yunnan Province Major Science and Technology Project (2017ZF007); National Natural Science Foundation of China (NSFC) (U1702282). The funders have no role in the design, collection, analysis, interpretation of data and in writing the manuscript of the study.

\section{Availability of data and materials}

The datasets generated and/or analysed during the current study are available in the NCBI database repository by the SRA accession: SRP151653, [https://www.ncbi.nlm.nih.gov/sra?linkname=bioproject_sra_all\&from_uid= 478287].

\section{Ethics approval and consent to participate}

The sample collections were performed in accordance with relevant guidelines and regulations approved by Ethical Committee of Institute of Medical Biology, Chinese Academy of Medical Sciences and Peking Union Medical College. All experimental procedures were approved by the Ethics Review Committee [Institutional Review Board (IRB)] of Institute of Medical Biology, Chinese Academy of Medical Sciences and Peking Union Medical College.

\section{Consent for publication}

Not applicable.

\section{Competing interests}

The authors declare that they have no competing interests.

\section{Author details}

${ }^{1}$ Center of Tree Shrew Germplasm Resources, Institute of Medical Biology, Chinese Academy of Medical Sciences and Peking Union Medical College, Yunnan Key Laboratory of Vaccine Research and Development on Severe Infectious Diseases, Yunnan Innovation Team of Standardization and Application Research in Tree Shrew, Zhao zong Road 66, Kunming 650118, China. ${ }^{2}$ Department of Acute Infectious Diseases Control and Prevention, Yunnan Provincial Centre for Disease Control and Prevention, Kunming 650022, China.

Received: 15 October 2018 Accepted: 27 August 2019

Published online: 02 September 2019

\section{References}

1. Lu C, Sun X, Li N, Wang W, Kuang D, Tong P, Han Y, Dai J. CircRNAs in the tree shrew (Tupaia belangeri) brain during postnatal development and aging. Aging (Albany NY). 2018;10(4):833-52.

2. Petry HM, Bickford ME. The second visual system of the tree shrew. J Comp Neurol. 2018;527(3):679-93.

3. Xiao J, Liu R, Chen CS. Tree shrew (Tupaia belangeri) as a novel laboratory disease animal model. Zool Res. 2017;38(3):127-37.

4. Ye L, He M, Huang Y, Zhao G, Lei Y, Zhou Y, Chen X. Tree shrew as a new animal model for the study of lung cancer. Oncol Lett. 2016;11(3):2091-5.

5. Feng Y, Feng YM, Lu C, Han Y, Liu L, Sun X, Dai J, Xia X. Tree shrew, a potential animal model for hepatitis $C$, supports the infection and replication of HCV in vitro and in vivo. J Gen Virol. 2017;98(8):2069-78.

6. Wang Z, Yi X, Du L, Wang H, Tang J, Wang M, Qi C, Li H, Lai Y, Xia W, et al. A study of Epstein-Barr virus infection in the Chinese tree shrew (Tupaia belangeri chinensis). Virol J. 2017:14(1):193.

7. Hu YD, Zhao Q, Zhang XR, Xiong LL, Zhang ZB, Zhang P, Zhang RP, Wang TH. Comparison of the properties of neural stem cells of the hippocampus in the tree shrew and rat in vitro. Mol Med Rep. 2018;17(4):5676-83.

8. Wu X, Chang Q, Zhang Y, Zou X, Chen L, Zhang L, Lv L, Liang B. Relationships between body weight, fasting blood glucose concentration, sex and age in tree shrews (Tupaia belangeri chinensis). J Anim Physiol Anim Nutr (Berl). 2013;97(6):1179-88. 
9. Fang $H$, Sun YJ, Lv YH, Ni RJ, Shu YM, Feng XY, Wang Y, Shan QH, Zu YN, Zhou JN. High activity of the stress promoter contributes to susceptibility to stress in the tree shrew. Sci Rep. 2016;6:24905.

10. Fuchs E, Flugge $G$, Ohl F, Lucassen P, Vollmann-Honsdorf GK, Michaelis T. Psychosocial stress, glucocorticoids, and structural alterations in the tree shrew hippocampus. Physiol Behav. 2001;73(3):285-91.

11. Fan Y, Huang ZY, Cao CC, Chen CS, Chen YX, Fan DD, He J, Hou HL, Hu L, $\mathrm{Hu} X \mathrm{X}$, et al. Genome of the Chinese tree shrew. Nat Commun. 2013;4:1426.

12. Wu M, Kuang DX, Huang YQ, Miao YR, Liu XC, Dai JJ. Age-related changes of corneal endothelial cell in healthy Chinese tree shrew measured by noncontact specular microscope. Int J Ophthalmol. 2017;10(12):1798-804.

13. $\mathrm{Xu} \mathrm{L}$, Chen SY, Nie WH, Jiang XL, Yao YG. Evaluating the phylogenetic position of Chinese tree shrew (Tupaia belangeri chinensis) based on complete mitochondrial genome: implication for using tree shrew as an alternative experimental animal to primates in biomedical research. J Genet Genomics. 2012;39(3):131-7.

14. Gaulke CA, Arnold HK, Humphreys IR, Kembel SW, O'Dwyer JP, Sharpton TJ. Ecophylogenetics clarifies the evolutionary association between mammals and their gut microbiota. MBio. 2018:9(5):1-14.

15. Sharpton TJ. Role of the gut microbiome in vertebrate evolution. mSystems. 2018;3(2):1-5.

16. Liu R, Hong J, Xu X, Feng Q, Zhang D, Gu Y, Shi J, Zhao S, Liu W, Wang X, et al. Gut microbiome and serum metabolome alterations in obesity and after weight-loss intervention. Nat Med. 2017;23(7):859-68.

17. Nicholson JK, Holmes E, Kinross J, Burcelin R, Gibson G, Jia W, Pettersson S. Host-gut microbiota metabolic interactions. Science. 2012;336(6086):1262-7.

18. Amato KR, Leigh SR, Kent A, Mackie Rl, Yeoman CJ, Stumpf RM, Wilson BA, Nelson KE, White BA, Garber PA. The role of gut microbes in satisfying the nutritional demands of adult and juvenile wild, black howler monkeys (Alouatta pigra). Am J Phys Anthropol. 2014;155(4):652-64.

19. Ren T, Grieneisen LE, Alberts SC, Archie EA, Wu M. Development, diet and dynamism: longitudinal and cross-sectional predictors of gut microbial communities in wild baboons. Environ Microbiol. 2016;18(5):1312-25.

20. Ley RE, Hamady M, Lozupone C, Turnbaugh PJ, Ramey RR, Bircher JS, Schlegel ML, Tucker TA, Schrenzel MD, Knight R, et al. Evolution of mammals and their gut microbes. Science. 2008;320(5883):1647-51.

21. Kang E, Crouse A, Chevallier L, Pontier SM, Alzahrani A, Silue N, CampbellValois FX, Montagutelli X, Gruenheid S, Malo D. Enterobacteria and host resistance to infection. Mamm Genome. 2018;29(7-8):558-76.

22. Li R, Xu W, Wang Z, Liang B, Wu JR, Zeng R. Proteomic characteristics of the liver and skeletal muscle in the Chinese tree shrew (Tupaia belangeri chinensis). Protein Cell. 2012;3(9):691-700.

23. Veit J, Bhattacharyya A, Kretz R, Rainer $G$. On the relation between receptive field structure and stimulus selectivity in the tree shrew primary visual cortex. Cereb Cortex. 2014;24(10):2761-71.

24. Yao YG. Creating animal models, why not use the Chinese tree shrew (Tupaia belangeri chinensis)? Zool Res. 2017;38(3):118-26.

25. Clayton JB, Gomez A, Amato K, Knights D, Travis DA, Blekhman R, Knight $R$, Leigh $S$, Stumpf $R$, Wolf $T$, et al. The gut microbiome of nonhuman primates: lessons in ecology and evolution. Am J Primatol. 2018;80(6):e22867.

26. Costello EK, Gordon Jl, Secor SM, Knight R. Postprandial remodeling of the gut microbiota in Burmese pythons. ISME J. 2010;4(11):1375-85.

27. Liao F, Gu W, Li D, Liang J, Fu X, Xu W, Duan R, Wang X, Jing H, Dai J. Characteristics of microbial communities and intestinal pathogenic bacteria for migrated Larus ridibundus in Southwest China. Microbiologyopen. 2018; 8(4):e00693.

28. Waite DW, Vanwonterghem I, Rinke C, Parks DH, Zhang Y, Takai K, Sievert SM, Simon J, Campbell BJ, Hanson TE, et al. Comparative genomic analysis of the class Epsilonproteobacteria and proposed reclassification to Epsilonbacteraeota (phyl. nov.). Front Microbiol. 2017;8:682.

29. Goto K, Ohashi H, Takakura A, Itoh T. Current status of helicobacter contamination of laboratory mice, rats, gerbils, and house musk shrews in Japan. Curr Microbiol. 2000;41(3):161-6.

30. Whary MT, Fox JG. Detection, eradication, and research implications of helicobacter infections in laboratory rodents. Lab Anim (NY). 2006;35(7): 25-27, 30-26

31. Larsen N, Vogensen FK, van den Berg FW, Nielsen DS, Andreasen AS, Pedersen BK, Al-Soud WA, Sorensen SJ, Hansen LH, Jakobsen M. Gut microbiota in human adults with type 2 diabetes differs from non-diabetic adults. PLoS One. 2010;5(2):e9085.
32. Gordon DM, FitzGibbon F. The distribution of enteric bacteria from Australian mammals: host and geographical effects. Microbiology. 1999; 145(Pt 10):2663-71.

33. Drzewiecka D. Significance and roles of Proteus spp. bacteria in natural environments. Microb Ecol. 2016;72(4):741-58.

34. Klindworth A, Pruesse E, Schweer T, Peplies J, Quast C, Horn M, Glockner FO. Evaluation of general 165 ribosomal RNA gene PCR primers for classical and next-generation sequencing-based diversity studies. Nucleic Acids Res. 2013; 41(1):e1.

35. Caporaso JG, Kuczynski J, Stombaugh J, Bittinger K, Bushman FD, Costello EK, Fierer N, Pena AG, Goodrich JK, Gordon Jl, et al. QIIME allows analysis of high-throughput community sequencing data. Nat Methods. 2010;7(5):335-6.

36. Edgar RC. UPARSE: highly accurate OTU sequences from microbial amplicon reads. Nat Methods. 2013;10(10):996-8.

37. Cao Y, Zheng X, Li F, Bo X. mmnet: an R package for metagenomics systems biology analysis. Biomed Res Int. 2015:2015:167249.

38. Quast C, Pruesse E, Yilmaz P, Gerken J, Schweer T, Yarza P, Peplies J, Glockner FO. The SILVA ribosomal RNA gene database project: improved data processing and web-based tools. Nucleic Acids Res. 2013;41(Database issue):D590-6.

39. Wilkinson TJ, Huws SA, Edwards JE, Kingston-Smith AH, Siu-Ting K, Hughes $M$, Rubino F, Friedersdorff M, Creevey CJ. CowPI: a rumen microbiome focussed version of the PICRUSt functional inference software. Front Microbiol. 2018;9:1095.

40. Wang X, Cui Z, Jin D, Tang L, Xia S, Wang H, Xiao Y, Qiu H, Hao Q, Kan B, et al. Distribution of pathogenic Yersinia enterocolitica in China. Eur J Clin Microbiol Infect Dis. 2009;28(10):1237-44.

41. Guo Y, Zhou H, Qin L, Pang Z, Qin T, Ren H, Pan Z, Zhou J. Frequency, antimicrobial resistance and genetic diversity of Klebsiella pneumoniae in food samples. PLoS One. 2016;11(4):e0153561.

42. Adesoji AT, Ogunjobi AA, Olatoye IO, Call DR. Prevalence of tetracycline resistance genes among multi-drug resistant bacteria from selected water distribution systems in southwestern Nigeria. Ann Clin Microbiol Antimicrob. 2015:14:35.

43. Bradford PA. Extended-spectrum beta-lactamases in the 21st century: characterization, epidemiology, and detection of this important resistance threat. Clin Microbiol Rev. 2001;14(4):933-51 table of contents.

44. Sutcliffe J, Grebe T, Tait-Kamradt A, Wondrack L. Detection of erythromycinresistant determinants by PCR. Antimicrob Agents Chemother. 1996;40(11): 2562-6.

45. Teng LJ, Hsueh PR, Ho SW, Luh KT. High prevalence of inducible erythromycin resistance among Streptococcus bovis isolates in Taiwan. Antimicrob Agents Chemother. 2001:45(12):3362-5.

46. Pfaller MA, Mujeeb I, Hollis RJ, Jones RN, Doern GV. Evaluation of the discriminatory powers of the dienes test and ribotyping as typing methods for Proteus mirabilis. J Clin Microbiol. 2000;38(3):1077-80.

47. Zhang SX, Zhou YM, Tian LG, Chen JX, Tinoco-Torres R, Serrano E, Li SZ, Chen $\mathrm{SH}, \mathrm{Ai} \mathrm{L}$, Chen JH, et al. Antibiotic resistance and molecular characterization of diarrheagenic Escherichia coli and non-typhoidal Salmonella strains isolated from infections in Southwest China. Infect Dis Poverty. 2018;7(1):53.

\section{Publisher's Note}

Springer Nature remains neutral with regard to jurisdictional claims in published maps and institutional affiliations.

Ready to submit your research? Choose BMC and benefit from:

- fast, convenient online submission

- thorough peer review by experienced researchers in your field

- rapid publication on acceptance

- support for research data, including large and complex data types

- gold Open Access which fosters wider collaboration and increased citations

- maximum visibility for your research: over $100 \mathrm{M}$ website views per year

At $\mathrm{BMC}$, research is always in progress.

Learn more biomedcentral.com/submissions 